\title{
INTERACTIVE MODELLING OF MPEG-4 DEFORMABLE HUMAN BODY MODELS
}

\author{
Hyewon Seo, Frederic Cordier, Laurent Philippon, Nadia Magnenat-Thalmann
} MIRALab, University of Geneva, CH-1211 Geneva, Switzerland

Key words: Deformation, simplification, MPEG-4 BDP, seamless body, animation ready model.

Abstract: Acquisition of various human body models is useful in many cases. In this paper, we present some of our recent work on body creation tools. Our goal is to enable rapid creation of various body models that are immediately usable for animation. In doing so, we aim to carry out realistic deformations on the human body models as well as make its usage simple. Our system is composed of several modules: (1) Skin attachment to an H-Anim skeleton is carried out first in order to get deformation in skeletal shape modification as well as in animation. (2) Volumetric deformation module deals with the volumetric scale of body parts such as breast, belly and bottoms. These deformation operators, together with the skeletal deformation allow the automatic adaptation of the body model to different sizes and proportions to accommodate anthropometrical variations. (3) Surface optimization is used to simplify the model in consideration of not only geometric features but also the animation aspect of it. (4) Finally, the BDP generation module describes the geometry of the model as well as how to animate it according to the MPEG-4 BDP specifications.

\section{INTRODUCTION}

In recent years, human characters have become more and more important in computer animation, virtual reality, entertainment, e-commerce and many other areas. Amongst many of the relevant techniques, model acquisition of the human character model has been attracted considerable attention from 
many researchers. In practice, it is the first step of the pipeline that encompasses addition of props, face and body animation, coordination along with stages or virtual environments, and synchronization with other media such as sound.

In general, human character modeling techniques are classified by the creative approach and the reconstructive approach. A variety of modeling methods, such as plaster modelling [11], sculptor [3], meatballs [14], freeform deformations [1] fall into the former approach. Within these methods, we often differentiate them according to whether they deal with the skeleton and the skin surface only (surface model) or they contain intermediate layers which simulate the muscle, bone, fat tissue, etc. (multi-layered model). While allowing an interactive design of human bodies either from scratch or by modify existing model, they however require considerable user intervention and thus suffer from a relatively slow production time and a lack of efficient control facilities.

Lately, much work has been devoted to the reconstructive approach. Some of them rely on stereo [4, 16], structured light [11] or 3D scanners [10]. Some systems use 2D images either from video sequences [5] or from photos $[6,7,8,9]$. In the latter case, modifying existing model tends to be popular due to the expenses of recovering 3D geometry. Based on adding details or features to an existing generic model, these approaches concern mainly the individualized shape and visual realism using a high quality textures. While they are effective and visually convincing in the cloning aspect, these approaches hardly give any control to the user; i.e., it is very difficult to modify these meshes to a different shape as the user intends.

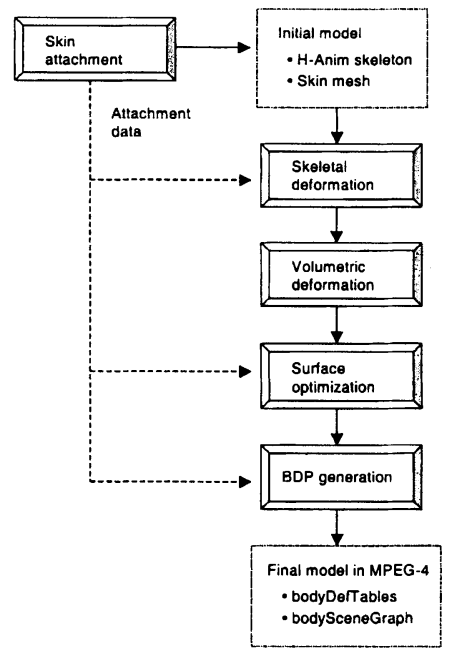

Figure 1. Overview of the creation process 
In this paper, we present our approach to create various human body models that are immediately usable for animation. A number of deformers are introduced for each part of the body to automatically adapt the body model to different sizes and proportions. We also discuss geometric optimization and standard support aspects of our system. Our system is composed of several modules working in a pipeline. Figurel gives an overview of the pipeline.

The rest of this paper is organized as follows: Our design specification is briefly introduced in Section 2. Section 3 describes the generic body model we have chosen to use. Section 4 explains the skeletal deformation used to achieve the variation of limb lengths of the body model. Section 5 details deformations used to modify the shape and volume of different parts of the body. The optimization and standard support issues will be briefly discussed in Section 6 and Section 7 respectively. Section 8 concludes this paper and describes our plans for future work.

\section{DESIGN SPECIFICATION}

Human morphology has a high variability depending on gender, age, occupation, etc. The description of the body can be defined by many ways. In this work, we focus on the structural measures or static size of the body such as height, breadth and width. We have chosen a set of anthropometrical measurements that correspond to the industrial standards:

- Size of the feet, hands, legs, arms and the neck.

- Width of legs, arms and the neck.

- Width of the waist, breast and hips.

Apart from the shape design, we aim to feature the system with the following aspects:

- Various levels of control: The user can simply type the measured values to drive automatic adaptation on the body or interactively add details.

- Animation ready model: Generate not only the geometric model but also information on how to deform it.

- Performance: Optimize the surface geometry with its deformation aspect considered.

- Standard support: Describe the resulting body model and animation information in MPEG-4 BDP format. 


\section{BODY MODEL}

We have worked on a generic model, which is composed of a skin mesh and a skeleton. For the skin surface mesh, we use body models that have been developed at EPFL and University of Geneva [14].

The skeleton hierarchy we have chosen to use is H-Anim Level of Articulation(LoA) 2 one [15]. This is important, as one of our goals is to make the resulting models MPEG-4 compatible. Figure2 illustrates the generic model for women and skeleton hierarchy excluding hand joints. Including the hierarchy or skeletal description of the body brings two practical advantages. Firstly, it enables an intuitive way of skeletal deformation. Skeletal deformation will be discussed later in this paper (Section 4). Secondly, this information is essential to define the animation ready body. The description of the body into MPEG-4 format (BDP) is described in Section 7.
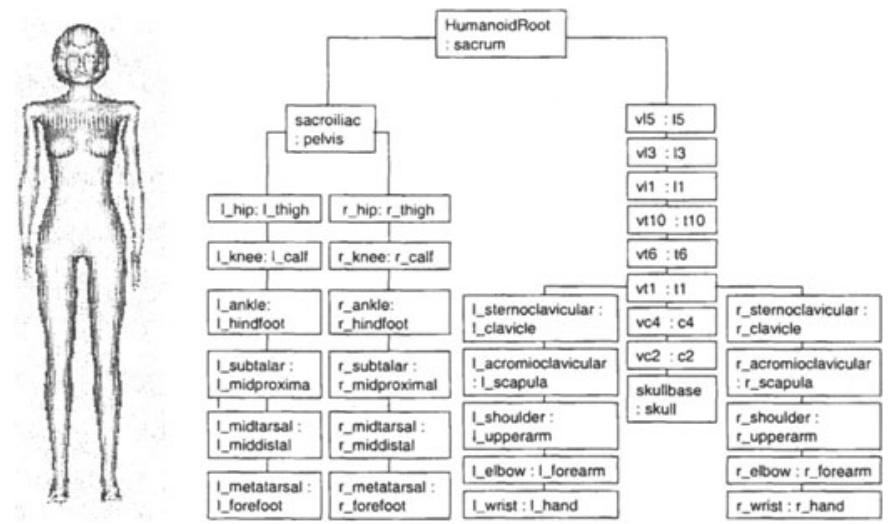

Figure 2. Generic body model: The skin surface model for a woman(left). The skeleton hierarchy excluding hands(right)

\section{SKELETAL DEFORMATION}

\subsection{Skin Attachment}

A proper skin attachment is essential to skeletal deformation as well as BDP exportation. The attachment is considered as assigning for each vertex of the mesh its affecting bones and corresponding weights. To say that a 
vertex is "weighted" with respect to a bone means that the vertex will move as the bone is rotated in order to stay aligned with it. At 100 percent weighting, for instance, the vertex follows the bone rigidly. This method combines for each vertex the transformation matrix of the bones in accordance to their weight. Using the attachment data, the position $\boldsymbol{P}_{v}$ of the skin vertex $v$ is defined by

$$
P_{v}=\sum_{i}\left(M_{i} \cdot K_{i} \cdot O_{i}\right)
$$

where $M_{i}$ is the transformation matrix of $i$ th affecting bone, $K_{i}$ is its weight and the offset $U_{i}$ is the distance from $v$ to the bone.

In order to speed up this process, we have defined a generic attachment data that can be used for every other model. We have explored several existing tools for the attachment [18]. On top of the chosen attachment tool, we have developed an importer/exporter in order to fully automate this process by reusing the once-done generic skin attachment data.

\subsection{Skeletal Deformation}

Once the skin is properly attached to the skeleton, transformation of the bone automatically derives transformation of the skin mesh. We provide two different levels of control to the user: At the highest level, the user simply types in the measure of these parameters instead of tedious selection and manual deformation. These parameters are then translated into modifications on the skeleton; the size of each bone is adapted to the new measurements. In case the user wants detailed and direct control, s/he can manipulate the skeleton directly in the rendering window using mouse.

This is useful especially to change the length or volume of the limbs. Figure 3 shows the modification of the limb volume and length we obtained by skeletal deformation.
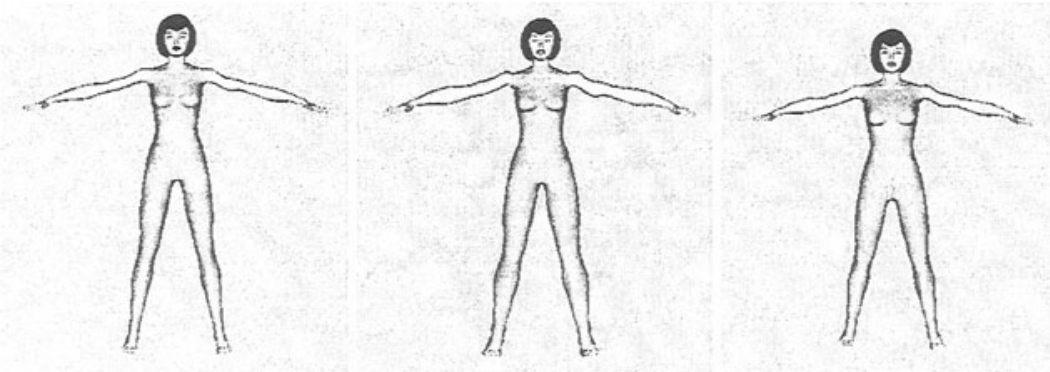

Figure 3. Skeletal deformation: Change of the limb volume and the limb length 


\section{VOLUMETRIC DEFORMATION}

For some parts of the body that concerns the volume measurements, deformation means more than simple transformation of associated bones. Breast or chest, belly, and hips are such examples. As the generic model is based on characteristic lines or contours and thus naturally forms a grid, we use parametric curves to deform these parts locally and smoothly.

\subsection{Breast}

Being represented as a regular grid, the breast region of the mesh forms a $20 \times 23$ array. The deformation takes place in the two directions as shown in Figure 4: one along the vertical direction and the other along the horizontal one.

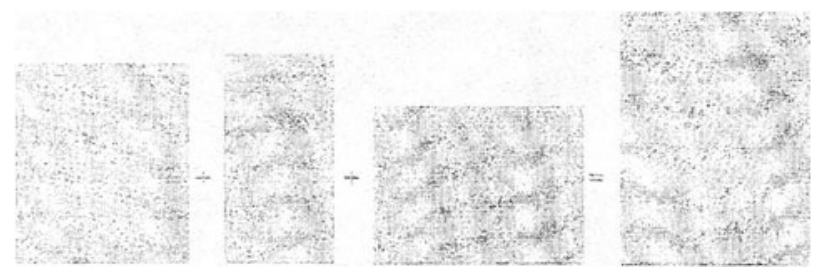

Figure 4. The deformation on the breast using NURBs

Along the vertical lines, we sensibly select 6 points as B-spline control points. The first and the last points are not moved in order not to create discontinuities on the surface. The second and the fifth point as well are just present to give a regular aspect to the surface, i.e. a curve that grows gradually. The third and the fourth point undergo a translation of factor $\mathrm{F}$, as shown in Figure 5. All the other points in the line are sampled using the Boor Cox algorithm.

Along the horizontal direction, the points should be located in such a way that it preserves the shape of the breast. The translations of the control points form a function $\mathrm{f}$, whose evolution takes the forms the shape of the breast. (See Figure 5.) In other words, the value of the factor $F$ will depend on the column for which the deformation is applied, multiplied by the degree of displacement desired by the user. Whenever the user increases or decreases the size via the user interface, s/he will have the resulting measurement value. Figure 6 illustrates some of the results we obtained. 


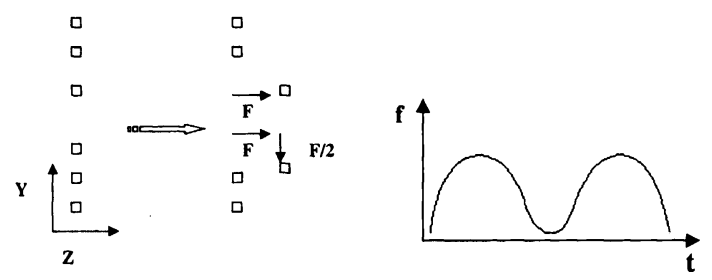

Figure 5. Translation of control points along the vertical direction (left) and function of displacement factor along the horizontal direction (right)

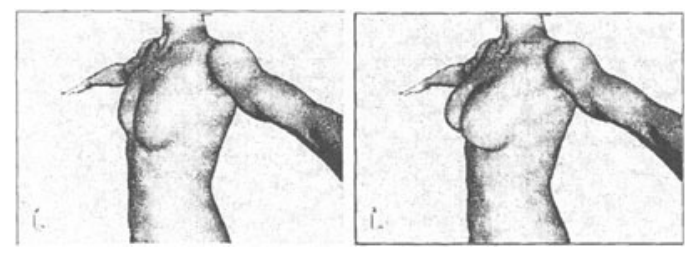

Figure 6. Deformation of the breast

\subsection{Belly}

The principle of the deformation for the belly is similar to that of the deformation for the breast except that we use Bézier curve in this case. We store the points of the body corresponding to the belly in a matrix of 20 by 25 . On the 20 points in the vertical direction, we select 4 points which will correspond to the control points of the Bézier curve (Figure 7).
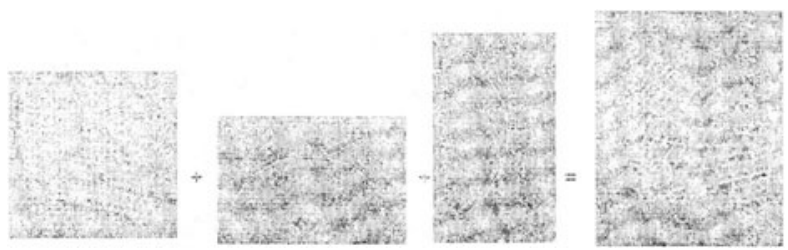

Figure 7. The belly deformation using Bézier

The two control points in the center are put forward along the $\mathrm{Z}$ axis by the factor F, coupled with a factor which corresponds to the position of the column the deformation is working. Once these 4 control points are placed at good positions, we sample the curve to position all the other points on it using the De Casteljau algorithm. The displacement factor along the 
horizontal direction takes the form of the belly as shown in Figure 8, which is followed by the resulting modified shapes in Figure 9.
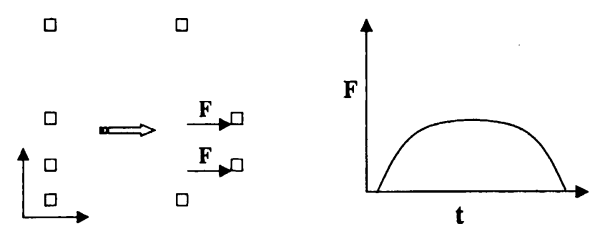

Figure 8. Displacement of control points (left) and function of displacement factor (right)

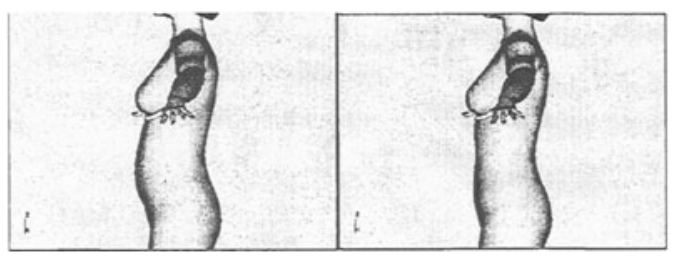

Figure 9. Deformation of the belly

\subsection{Bottom}

A simple method of deformation based on the FFD (Free Form Deformation) is used for the bottom. The bounding box of the bottom is regularly sampled to obtain $4 \times 4 \times 4=64$ control points of the Bézier volume. As we move any of the control points, the enclosed surface will be deformed accordingly.

In most of the cases, only certain points of the volume are necessary. We thus get the differently sized bottoms by simultaneously moving these points, which are the four central points of the back face of the Bézier volume.

\section{OPTIMIZATION}

Traditionally, simplification techniques have dealt mainly with static objects. These methods focus on preserving visually important features of the model such as sharp edges, high curvatures, and silhouettes during the simplification [2, 13]. When it comes to animated character models however, the direct use of these methods does not make much sense. Apart from the geometric characteristics of the surface, we need also to consider 
the animation aspect of the model or more specifically, the skin-to-bone attachment information. By assigning higher priority to those vertices that have more influencing bones, we can keep more vertices near joints so that the deformation during animation appears as natural as possible. In this work, we took a simple vertex decimation method with the following evaluation function:

$\operatorname{Eval}(\mathrm{v})=\mathrm{W}_{b} \times\left(\mathrm{W}_{0} \times\right.$ distance $+\mathrm{W}_{1} \times$ normalDeviation $+\mathrm{W}_{2} \times$ curvature $)$

where $\mathrm{W}_{b}$ is the number of bones which the vertex $\mathrm{v}$ is attached to, $\mathrm{W}_{0}$ is for the distance between the old vertex and the average plane of the simplified polygon, $\mathrm{W}_{l}$ for the normal deviations of a vertex and thus sharp features of the mesh, and $W_{2}$ for high curvature regions. Each of the weights, when it has a high value compared to the other weights, preserves different characteristics of the mesh.

Some of the results we obtained are shown in Figure 10.
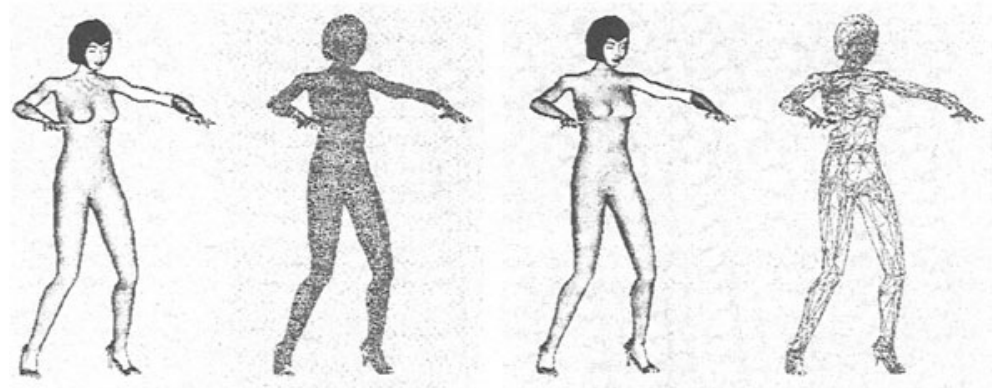

Figure 10. Different Levels of Detail description of the body surface: Full description with 13389 vertices and 25853 faces(left). Simplified model with 4726 vertices and 8578 faces (right).

\section{STANDARD SUPPORT}

This section explains our work to describe the body model according to the MPEG-4 BDP specifications. The 'Body' node in MPEG-4 organizes definition and animation of a body [17]. Our system in this frame mainly concerns the BDPs, which by definition (Figure 11) gives geometrical description of a body model along with the information on how to animate it. Here, the animation means a skin deformation when given a set of joint angles. 


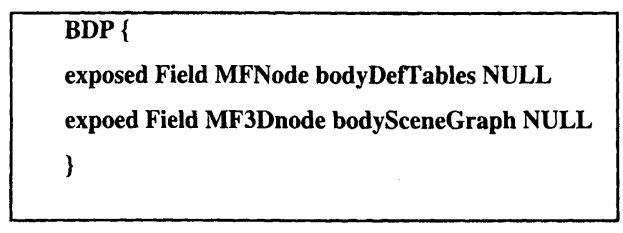

Figure 11. The BDP node definition in MPEG-4.

The 'bodySceneGraph' is strongly based on VRML H-Anim 1.1 specification [15]. The H-Anim specifies a standard way of representing humanoids in VRML97. The human body consists of a number of 'Segments', which are connected to each other by 'Joints'. The full H-Anim hierarchy is composed of 94 skeleton joints and 12 skin segments including the head, hands and feet.

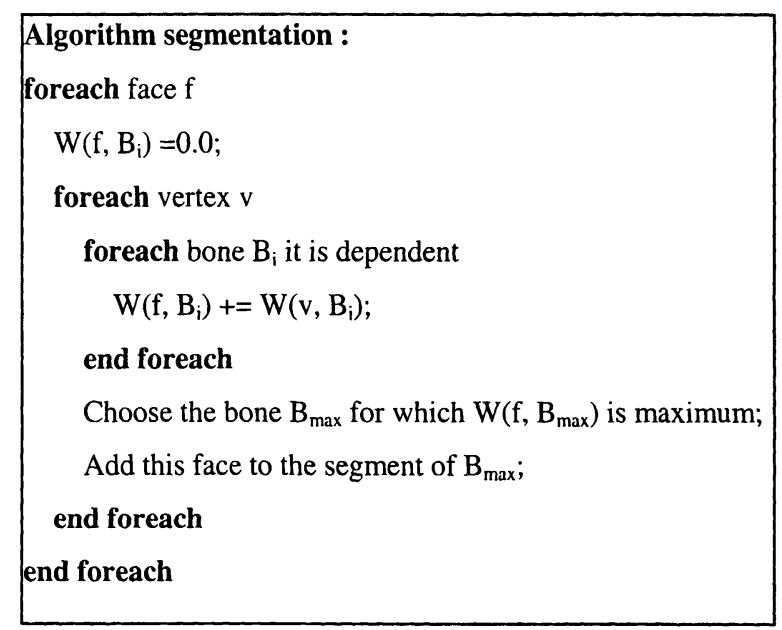

Figure 12. Segmentation algorithm

As mentioned earlier, our choice of the skeleton hierarchy is H-Anim compatible. The remaining work is to segment the skin mesh and locate each of them into the skeleton hierarchy as a proper child node of corresponding joint. Our segmentation algorithm is described in Figure 12. After the segmentation, each skin part is saved in the local coordinate system and is connected to a 'Joint' as a 'Segment' child node.

The bodyDefTables field defines how the segmented mesh of the Segment node in the bodySceneGraph is modified or deformed based on sets of BAPs. Each bodyDefTable contains a list of BAPs, and a list of vertices and their displacements in the bodySceneGraph that are affected by these BAPs. Following are the features of our bodyDefTables generator.

- A number of key postures are provided by default. 
- The user can then add a new posture or remove any of the registered postures.

- When confirmed, all the listed postures are transformed into sets of BAPs and the accordingly deformed segments into displacements.

\section{CONCLUSION AND FUTURE WORK}

In this paper, we have introduced our on-going work on a methodology for creating and scaling bodies and discussed various relevant issues. Our approach provides an efficient way of creating bodies and the resulting models are directly usable for web application. With its support for MPEG-4 which is an industrially recognized format, the resulting model enables efficient and immediate animation for various applications: virtual fashion try on, for instance.

Our plans for future work focus on ways to improve the quality of the model in terms of the accuracy and texture. We also plan to integrate our work with face models for further extensions into a crowd generation system.

The ultimate goal of this research is to enable rapid creation of various body models immediately usable for animation. By doing so, we would like to extend the area of human character modelling to encompass the animation, rendering performance, standard, and other various practical considerations.

\section{ACKNOWLEDGEMENTS}

This work is supported by SoNG, funded by the European Community.

\section{REFERENCES}

1. Chadwick, J., Haumann, D.R. and Parent, R.E., "Layerd Construction for Deformable Animated Characters", Computer Graphics, In Computer Graphics(Proc. SIGGRAPH '89), pp.234-243, ACM Press, 1989.

2. Cohen,J. Varshney,A., Manocha,D., Turk,G. and Weber,H., "Simplification Envelopes", In Computer Graphics(Proc. SIGGRAPH '96), pp.119-128, ACM Press, 1996.

3. DeRose T., Kass M., Truong T., "Subdivision Surfaces in Character Animation", In Computer Graphics (Proc. SIGGRAPH '98), ACM Press, pp. 85-94, 1998.

4. Devernay F., Faugeras O.D., "Computing Differential Properties of 3-D Shapes from Stereoscopic Images without 3-D Models", In Proc. Of Computer Vision and Pattern Recognition, pp.208-213, 1994. 
5. Fua P., "Human Modeling from Video Sequence", Geomatics Info Magazine, 13(7): 63-65, July 1999.

6. Gu J., Chang T., Mak I., Gopalsamy S., Shen H., and Yuen M., "A 3D Reconstruction System for Human Body Modeling”, In Modelling and Motion Capture Techniques for Virtual Environments (Proc. CAPTECH'98), pp.229-241, Springer, 1998.

7. Hilton A., Beresford D., Gentils T. and Smith R. and Sun W., "Virtual People: Capturing human models to populate virtual worlds". In Computer Animation (Proc. Computer Animation'99), pp.174-185, 1999.

8. Kakadiaris A. and Metaxas D., "3D Human Body Acquisition from Multiple views", In Proceedings of the Fifth ICCV, pp.618-623, 1995.

9. Lee W.-S., Gu J., Magnenat-Thalmann N., "Generating Animatable 3D Virtual Humans from Photographs", Proc. Eurographics 2000. pp.1-10, 2000.

10. Lee Y., Terzopoulos D., and Waters K., "Realistic Modeling for Facial Animation", In Computer Graphics (Proc. SIGGRAPH' 96), ACM Press, pp. 55-62, 1996.

11. Magnenat-Thalmann N., Thalmann D., "The direction of Synthetic Actors in the film Rendez-vous à Montreal", Computer Graphics and Applications, IEEE Computer Society Press, 7(12): 9-19, 1987.

12. Proesmans M., Van Gool L., "Reading between the lines - a method for extracting dynamic 3D with texture", In Proceedings of VRST, pp. 95-102, 1997.

13. Schroeder,W., Zarge, J. and Lorensen,W., "Decimation of Triangle Meshes", In Computer Graphics(Proc. SIGGRAPH '92), Volume 25, No. 3, pp.65-70, ACM Press, 1992.

14. Shen J., Thalmann D., "Interactive Shape Design Using Metaballs and Splines", In Proc. of Implicit Surfaces '95, Grenoble, pp.187-196.

15. Specification for a Standard Humanoid, Appendix A: Suggested Body Dimensions and Levels of Articulation. http://www.H-Anim.org

16. http://www.turing.gla.ac.uk/turing/copyrigh.htm

17. International Organization for Standardization Organization International Normalization ISO/IEC JTC 1/SC 29/WG 11. Coding of Moving Picture and Audio (N2739 subpart2), 1999.

18. Physic and Skin modifier, 3D Studio max, Kinetix. 